\title{
Characterization of LexA-mediated Transcriptional Enhancement of Bidirectional Hydrogenase in Synechocystis sp. PCC 6803 upon Exposure to Gamma Rays
}

\author{
Jin-Hong Kim,*, Min Hee Lee, Ji Hong Kim¹, Yu Ran Moon¹, Eun Ju Cho¹, Ji Eun Kim, \\ Choon-Hwan Lee ${ }^{2}$, and Byung Yeoup Chung ${ }^{1, *}$
}

${ }^{1}$ Advanced Radiation Technology Institute, Korea Atomic Energy Research Institute, Jeongeup 580-185, Korea

${ }^{2}$ Department of Molecular Biology, Pusan National University, Busan 609-735, Korea;

\begin{abstract}
Influence of gamma rays on the cyanobacterium Synechocystis sp. PCC 6803 cells was investigated in terms of a bidirectional hydrogenase, which is encoded by hoxEFUYH genes and responsible for biohydrogen production. Irradiated cells revealed a substantial change in stoichiometry of photosystems at one day after gamma irradiation at different doses. However, as evaluated by the maximal rate of photosynthetic oxygen evolution, maximal photochemical efficiency of photosystem II, and chlorophyll content, net photosynthesis or photosynthetic capacity was not significantly different between the control and irradiated cells. Instead, transcription of hoxE, hoxH, or lexA, which encodes a subunit of bidirectional hydrogenase or the only transcriptional activator, LexA, for hox genes, was commonly enhanced in the irradiated cells. This transcriptional enhancement was more conspicuously observed immediately after gamma irradiation. In contrast, hydrogenase activities were found to somewhat lower in the irradiated cells. Therefore, we propose that transcription of hox genes should be enhanced by gamma irradiation in a LexA-mediated and possibly photosynthesis-independent manner and that this enhancement might not induce a subsequent increase in hydrogenase activities, probably due to the presence of post-transcriptional and/or posttranslational regulatory mechanisms.
\end{abstract}

Synechocystis sp. PCC 6803 is a fresh-water, unicellular, non-nitrogen fixing cyanobacterium, containing a single bidirectional $\mathrm{NiFe}$-hydrogenase encoded by hoxEFUYH. ${ }^{1}$ Bidirectional hydrogenasessplit molecular hydrogen into protons and low potential electrons or combine protons and electrons to molecular hydrogen. Althoughthe physiological role of this enzyme in cyanobacteria remains unclear to date, it has been suggested that the enzyme may involve in fermentation, respiratory and photosynthetic electron flow. ${ }^{2}$

*To whom correspondence should be addressed. E-mail: jhongkim@kaeri.re.kr; by chumg@kaeri.re.kr Received January 4, 2012; accepted January 30, 2012
The cyanobacterial hydrogenase and hydrogen metabolism is controlled by environmental factors such as anaerobiosis, molecular hydrogen, nickel, ${ }^{3}$ and nitrate- and sulfurlimitations ${ }^{4,5}$ Recently, important clues about the genetic control of hox genes were obtained from Synechocystis sp. PCC 6803. LexA, a transcriptional repressor in the gene expression for the SOS response of Escherichia coli, was revealed to function as a transcriptional activator for the bidirectional hydrogenase genes, hoxEFUYH, in Synechocystis. ${ }^{1,26}$ The cyanobacterial LexA has two binding sites upstream of hoxEFUYH and is the first and only transcription regulator identified so far for expression of the bidirectional hydrogenase genes in cyanobacteria. It also has been suggested that LexA might be important for regulation of genes involved in carbon metabolism rather than the typical DNA repair genes in Synechocystis. ${ }^{7}$ Moreover, cyanobacteria are known to be much stronger to DNA damaging agents than Escherichia coli. Although LexA does not involve in activation of DNA repair genes in cyanobacterial cells, there exists a possibility for additional cellular responses and/or physiological functions of LexA to DNA damaging agents, e.g., ionizing radiation. The low sequence homology (about 28\%) of LexA between Escherichia coli K12 and Synechocystis sp. PCC 6803 allows this possibility. In the present study, therefore, we investigated whether gamma rays might work as an artificial elicitor for activation of the bidirectional hydrogenase genes. In addition, possible relationships among photosynthetic activity, transcript level of lexA and two hox genes, and hydrogenase activity, were assessed in Synechocystis sp. PCC 6803 cells after exposure to gamma rays at different doses.

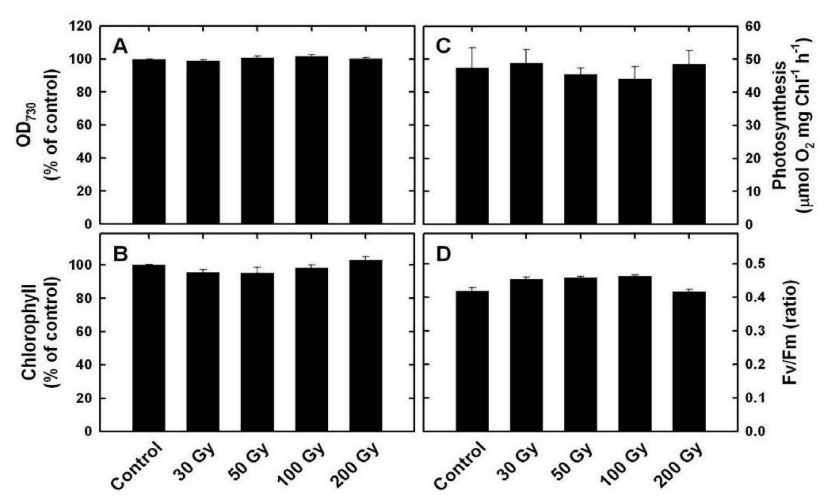


Figure 1. Growth rate (OD730, A), chlorophyll content (B), maximal rate of photosynthetic $\mathrm{O}_{2}$ evolution (C), and maximal photochemical efficiency of PSII (Fv/Fm, D) in the control and irradiated cells. Five-day-old cultures with an OD730 of 1.2-1.5 were exposed to gamma rays at a dose rate of $1,1.67,3.33$, or $6.67 \mathrm{~Gy} \mathrm{~min}^{-1}$ for $30 \mathrm{~min}$. Then the control and irradiated cells were harvested one day later for all measurements. Bars represent means \pm SE of $6-12$ measurements from 2-4 independent experiments.

Cyanobacteria have been known to be relatively resistant to DNA damaging agents, e.g., ionizing radiation. However, gamma rays as an artificial elicitor toenhance activation of the bidirectional hydrogenase genes, no or only minor effects on viability of cyanobacterial cells are a prerequisite for the sustainable biohydrogen production. Although the bidirectional hydrogenase modulates transcription of photosynthetic genes $p s b A, p s a A$ and petB, working as an electron valve during photosynthesis, ${ }^{8}$ growth, chlorophyll content, and net photosynthesis of Synechocystis cells were little affected by gamma rays up to 200 Gy (Figure 1A-C). Instead, the maximal photochemical efficiency of PSII, $\mathrm{Fv} / \mathrm{Fm}$, was slightly increased by gamma irradiation up to 100 Gy (Figure 1D). These results may imply that the cyanobacterium Synechosystisis overall tolerant to gamma rays up to $200 \mathrm{~Gy}$.

However, we cannot rule out the possibility that functional or structural changes in photosynthetic electron transport systems of Synechocystis cells were caused by gamma irradiation. To investigate a change in stoichiometry of photosystems constituting photosynthetic electron transport systems, therefore, relative amounts of photosystems I (PSI) to II (PSII) were determined in the control and irradiated cells by measuring $77 \mathrm{~K}$ fluorescence emission spectra with excitation at 440 or $580 \mathrm{~nm}$. The ratio of PSI to PSII can be expressed as a ratio of F725 to F695 or F720 to F690. ${ }^{9-11}$ It was found to be substantially higher in the irradiated cells than in the control, except for the 200-Gy cells (Figure 2A-E). Moreover, the $77 \mathrm{~K}$ fluorescence emission spectra upon excitation at $580 \mathrm{~nm}$ demonstrated that irradiation of Synechosystis cells with gamma rays up to 100 Gy decreased PSII emission at 680 to $690 \mathrm{~nm}$ more than PSI emission at $720 \mathrm{~nm}$ (Figure 2F-J). These results suggest thatthe structural changes of photosystems after gamma irradiation would be more conspicuous in PSII than in PSI and be associated with no significant decrease in photosynthetic capacity.

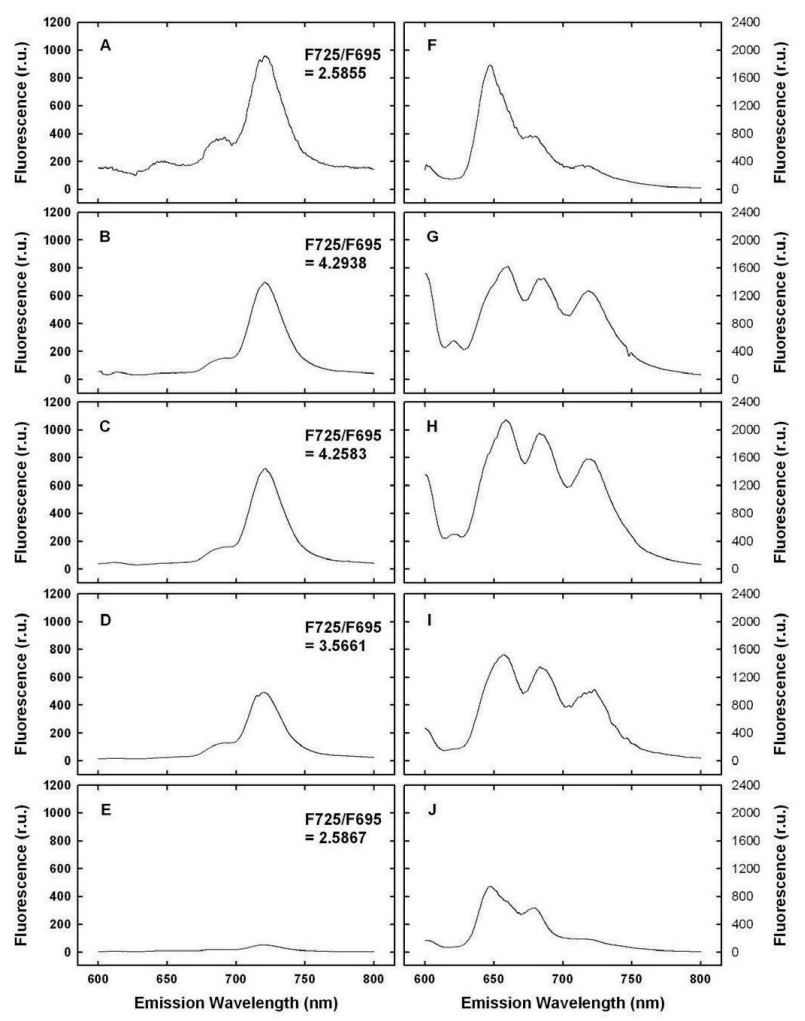

Figure 2. Low-temperature (77 K) emission spectra in the control and irradiated cells. A and F, control; B and G, irradiated (30 Gy); C and H, irradiated (50 Gy); D and I, irradiated (100 Gy); and Eand J, irradiated (200 Gy). Spectra were obtained as a relative fluorescence at the emission wavelengths of $600 \mathrm{~nm}$ to $800 \mathrm{~nm}$ with excitation at $440(\mathrm{~A}-\mathrm{E})$ or $580 \mathrm{~nm}(\mathrm{~F}-\mathrm{J})$, respectively. Mean values of three different measurements were used for each graph.

Upon treatment of Escherichia coli cells with DNA damaging agents such as ultraviolet light (UV) and mitomycin C, the proteolytic inactivation of LexA, a transcriptional repressor in the gene expression for the SOS response, is induced in association withactivated RecA molecules and then leads to increased synthesis of RecA for propagation of the SOS response. ${ }^{12}$ Since the lexA itself is repressed by its own product, LexA, its gene expression is also enhanced by the inducing treatments. In contrast, it has been recently reported in cyanobacterial cells that the Synechocystis lexA and recA promoters were strong and UV insensitive, thereby showing no correlation with the Escherichia colitype SOS response. ${ }^{7,13}$ 


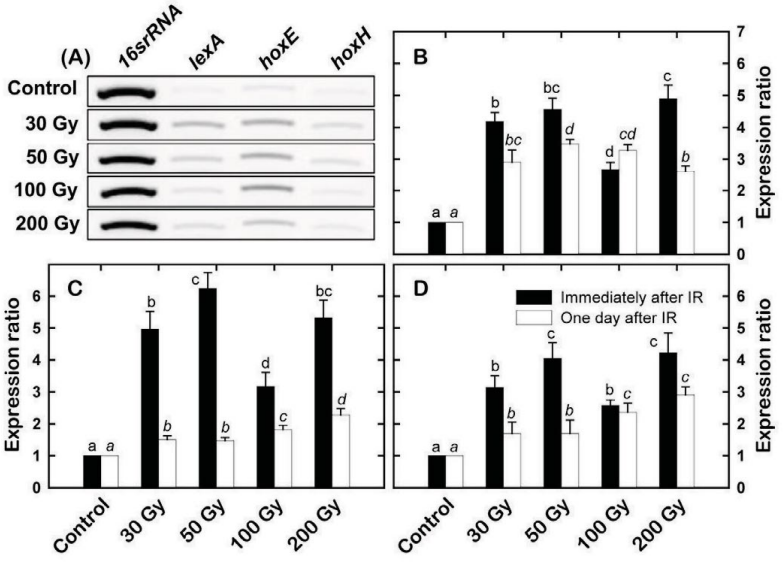

Figure 3. Expression of lexA, hoxE, and hoxH in the control and irradiated cells. A, RT-PCR; and B, C, and D, Real-time quantitative PCR for lexA, hoxE, and hoxH, respectively. 16 srRNA was used as an endogenous control gene to normalize for differences in the amounts of total RNA. In B$\mathrm{D}$, the expression ratio indicates the relative transcript levelof each gene, which was calculated by the comparative $\left(2^{-\Delta \Delta \mathrm{Ct}}\right)$ method. ${ }^{15}$ Bars represent means $\pm \mathrm{SE}$ of four measurements from two independent experiments. The mean values without a common letter are significantly different at $P=0.05$ by Tukey's honestly significant difference (HSD) test. IR, gamma-irradiation.

Unlike UV, when Synechocystis sp. PCC 6803 cells were irradiated with gamma rays of 50-200 Gy, the transcription level of lexA was 2.7- to 4.9-fold increased immediately with little dependence on the radiation dose (Figure 3A and B). Interestingly, a similar pattern was also observed in the transcript level of hoxE or hoxH, which was 3.2- to 6.2fold or 2.6- to 4.2-fold increased, respectively, immediately after gamma irradiation (Figure $3 \mathrm{~A}, \mathrm{C}$, and D). Even one day later, the transcript level of lexA, hoxE, or hoxH was 2.6- to 3.5-fold, 1.5 - to 2.3-fold, or 1.7- to 2.9-fold higher than that of the control, respectively. These results imply that the increase in transcription of hoxE and hoxH after gamma irradiation may occur through LexA, the only transcriptional activator for hoxEFUYH. Accordingly, the higher transcript level of hoxE than that of hoxH may be due to the relative positions of the two genes from the promoter in the Synechocystis hox operon. In contrast, it was also reported that Synechocystis LexA-related protein functions as a transcriptional repressor for cyanobacterial RNA helicase, $c r h R$, in a redox-responsive manner. ${ }^{13}$ Although Synechocystis LexA does not participate in regulation of DNA repair genes for the SOS response, it may be crucial in cell viability and transcription of hoxEFUYH after gamma irradiation. However, since our previous study revealed transcriptional activation of Synechocystis hox promoter in transformed Escherichia coli cells irrespective of LexA, ${ }^{14}$ it cannot be excluded that other unknown transcriptional regulators as well as LexA may involve in the enhanced transcription of hoxE and hoxH after gamma irradiation.
Next, to reveal the influence of the enhanced hoxE and hoxH transcription on production of biohydrogen, hydrogenase activities were investigated after gamma irradiation. The hydrogenase activities were found to be somewhat lower in the irradiated cells (Figure 4). Therefore, the increased transcript levels of hoxE and hoxH after gamma-irradiation didn't imply the subsequent enhancement of hydrogenase activity and biohydrogen production. Actually, SDS-PAGE analysis of total cell extracts showed that the levelsof HoxE and HoxH proteins were not significantly different between the control and irradiated cells (data not presented). In contrast, the decrease of hydrogenase activities in the irradiated cells might be due to incomplete enzyme maturation. Post-translational modifications via maturation enzymes, e.g., HypA-F and HoxW proteins, are known to be necessarily required for full activation of nascent cyanobacterial hydrogenases. ${ }^{16}$ Taken together, the data shown in Figures 3 and 4 suggest that the hydrogenase activities could be rather inhibited in spite of the enhanced transcription of hoxE and hoxH after gamma irradiation.

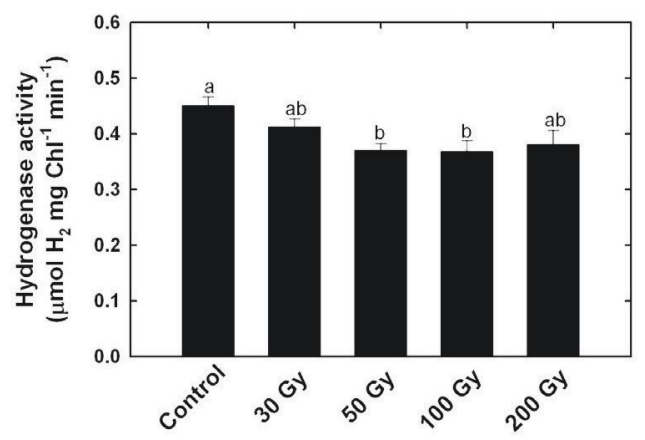

Figure 4. Hydrogenase activity in the control and irradiated cells. Hydrogenase activities were measured in the dark by adding methyl viologen and dithionite as artificial electron donors. Bars represent means \pm SE of six measurements from three independent experiments. The mean values without a common letter are significantly different at $P=$ 0.05 by Tukey's honestly significant difference (HSD) test.

In conclusion, we demonstrated that the substantial change in stoichiometry of Synechocystis photosystems within one day after gamma irradiation did not cause significant damages in photosynthetic capacity and that gamma irradiation could increase transcription of hox genes in a LexA-mediated and possibly photosynthesis-independent manner. Since the gamma-ray-induced enhancement of hox gene transcription was null for hydrogenase activities, the presence of post-transcriptional and/or posttranslational regulatory mechanisms was suggested for the failure of a subsequent increase in hydrogenase activities. Therefore, these regulatory mechanisms involving in hydrogenase activities after gamma irradiation will be further studied for enhancement of biohydrogen production. 
KEYWORDS: Bidirectional hydrogenase, Biohydrogen, Gamma rays, Synechocystis

\section{ACKNOWLEDGEMENT}

This research was supported by the Nuclear R \& D Program of the Ministry of Education, Science and Technology (MEST), Republic of Korea.

\section{SUPPORTING INFORMATION}

Experimental procedures are available free of charge via the Internet at http://photos.or.kr.

\section{REFERENCES AND NOTES}

1. Antal, T. K.; Oliveira, P.; Lindblad, P. Int. J. Hydrogen Energ. 2006, 31, 1439-1444.

2. Gutekunst, K.; Phunpruch, S.; Schwarz, C.; Schuchardt, S.; Schulz-Friedrich, R.; Appel, J. Mol. Microbiol. 2005, 58, 810-823.

3. Axelsson, R.; Lindblad, P. Appl. Environ. Microbiol. 2002, 68, 444-447.

4. Sheremetieva, M.; Troshina, O.; Serebryakova, L. T.; Lindblad, P. FEMS Microbiol. Lett. 2002, 214, 229-233.

5. Antal, T. K.; Oliveira, P.; Lindblad, P. J. Appl. Microbiol. 2005, 98, 114-120.

6. Oliveira, P.; Lindblad, P. FEMS Microbiol. Lett. 2005, 251, 59-66.

7. Domain, F.; Houot, L.; Chauvat, F.; Cassier-Chauvat, $C$. Mol. Microbiol. 2004, 53, 65-80.

8. Appel, J.; Phunpruch, S.; Steinm?ller, K.; Schulz. R. Arch. Microbiol. 2000, 173, 333-338.

9. Murakami, A. Photosynth. Res. 1997, 53, 141-148.

10. Song, E. K.; Zulfugarov, I. S.; Kim, J.-H.; Kim, E. H.; Lee, W. S.; Lee, C.-H. J. Plant Biol. 2004, 47, 289-299.

11. Gutthann, F.; Egert, M.; Marques, A.; Appel, J. Biochim. Biophys. Acta 2007, 1767, 161-169.

12. Miki, T.; Shirabe, K.; Ebina, Y.; Nakazawa, A. Nucleic Acids Res. 1984, 12, 1203-1217.

13. Patterson-Fortin, L. M.; Colvin, K. R.; Owttrim, G. W. Nucleic Acids Res. 2006, 34, 3446-3454.

14. Moon, Y. R.; Lee, M. H.; An, B. C.; Chung, B. Y.; Kim, J.S.; Park, Y.-I.; Kim, C. S.; Kim, J.-H. J. Radiat. Ind. 2009, 3, 107-114.

15. Schmittgen, T. D.; Zakrajsek, B. A.; Mills, A. G.; Gorn, V.; Singer, M. J.; Reed, M. W. Anal. Biochem. 2000, 285, 194-204.

16. Tamagnini, P.; Leit ão, E.; Oliveira, P.; Ferreira, D.; Pinto, F.; Harris, D. J.; Heidorn, T.; Lindblad, P. FEMS Microbiol. Rev. 2007, 31, 692-720. 